\title{
Evaluating the Chrono-pharmacology of Icosapent Ethyl to Assess its Therapeutic Efficacy in Reducing Hypertriglyceridemia Associated Cardiovascular Events
}

\author{
Arun HS Kumar ${ }^{1, *}$, Melvin George $e^{2,3}$
}

\begin{abstract}
Background: The pharmacological efficacy of drugs used to treat chronic diseases is evaluated based on a cumulative performance over a fixed term (usually 3 to 5 years). The justification for selecting such fixed terms is scientifically not convincing especially considering patients will be taking such drugs for a much longer time frame. Hence to address this gap we present here a novel concept of chrono pharmacological assessment of drug efficacy. Materials and Methods: Using the data reported from the recently concluded REDUCE-IT trail evaluating the pharmacological efficacy of Icosapent Ethyl to reduce the cardiovascular adverse effects in patients with elevated triglyceride levels, we compared the year wise slopes of the curves in the placebo and Icosapent Ethyl administered group as an approach of chrono pharmacological assessment of drug efficacy. Results: The chrono pharmacological assessment revealed an unexpected observation of increased rate of adverse events compared to the placebo group in the years 3 to 4 and post year 5 of the Icosapent Ethyl administered group. Such increased rate of adverse events may perhaps be due to the cumulative effects of the drug. Conclusion: This study highlights the merit of chrono pharmacological assessment in identifying any potential adverse event rates with chronic use of drugs. Hence chrono pharmacological evaluation of drugs used to treat chronic diseases must be considered to improve drug dosage regimen and achieve optimal therapeutic efficacy, safety and cost effectiveness.
\end{abstract}

Key words: Drug safety, Chrono pharmacology, Pharmacoeconomics, Drug dosage, Therapeutic efficacy.

\section{INTRODUCTION}

Elevated triglyceride levels remain a significant risk factor among patients with cardiovascular diseases despite being on the recommended optimal medical therapy. ${ }^{1-3}$ Previous attempts to reduce the elevated triglycerides levels using treatments such as fibrates, niacin, docosahexaenoic acid, long chain fatty acid products or Eicosapentaenoic Acid (EPA) without or with statins have shown to minimally or moderately reduce rate of adverse cardiovascular events.-9 Among these triglyceride lowering agents (Figure 1), EPA has shown the most pharmacological efficacy by $20 \%$ reduction in myocardial infarction and associated coronary events. ${ }^{7-11}$ Based on the promising outcome from EPA therapy, very recently a highly purified and stable ethyl ester of EPA was tested for pharmacological efficacy in the Reduction of Cardiovascular Events with Icosapent Ethyl Intervention Trial (REDUCE-IT, ClinicalTrials.gov number, NCT01492361)..$^{7-9,12}$

Icosapent Ethyl is a very interesting chemical. Besides its ability to reduce triglyceride levels, it is also reported to have antioxidant, anti-inflammatory and membrane/atherosclerotic plaque stabilization properties. ${ }^{9-11,13,14}$ In the REDUCE-IT trial patients

Cite this article : Kumar AHS, George M. Evaluating the Chrono-pharmacology of Icosapent Ethyl to Assess its Therapeutic Efficacy in Reducing Hypertriglyceridemia Associated Cardiovascular Events. BEMS Reports. 2019;5(2):26-8. were put on $2 \mathrm{~g}$ of Icosapent Ethyl twice daily with food and were followed up for 5 years. ${ }^{7,9}$ A significant reduction in mortality and ischemic cardiovascular events was observed among the patients who received Icosapent Ethyl. ${ }^{7,9,15}$ Considering the promising outcome from the REDUCE-IT trial Icosapent Ethyl is now been recommended for the reduction of cardiovascular risk management in patients with elevated triglyceride levels. ${ }^{1,15,16}$

The pharmacological approach in the management of chronic diseases by default involves patients being on the medication indefinitely. ${ }^{16-19}$ Although changes to the medication is considered in the events of any undesirable effects. ${ }^{19-21}$ Chronic consumption of the medicines does lead to its progressive accumulation in several tissue niche and the pharmacokinetics and pharmacodynamics of such tissue specific accumulation of medicine is rarely investigated clinically probably due to lack of suitable protocols to investigate such impact. Using the data available on Icosapent Ethyl from the REDUCE-IT trial, this study evaluates the chronological pharmacodynamics of Icosapent Ethyl with an aim to assess if it can help to improve the therapeutic efficacy of Icosapent Ethyl.

\section{Arun HS Kumar ${ }^{1, *}$, Melvin George $^{2,3}$}

'Department of Veterinary Biosciences, School of Veterinary Medicine, University College Dublin, Belfield, Dublin, IRELAND.

${ }^{2}$ Department of Clinical Pharmacology, SRM Medical College Hospital and Research Centre, Kattankulathur, Chengalpattu, Tamil Nadu, INDIA. ${ }^{3}$ Consultant Clinical Research, Hindu Mission Hospital, Chennai, Tamil Nadu, INDIA.

\section{Correspondence}

Dr. Arun HS Kumar, DVM,

Department of Veterinary Biosciences, Director Stemcology, School of Veterinary Medicine, University College Dublin, Belfield, Dublin-04, IRELAND.

Ph.no: 00-353-17166230

Email: arun.kumar@ucd.ie

History

- Submission Date: 09-10-2019;

- Review completed: 29-10-2019;

- Accepted Date: 03-11-2019.

DOI : 10.5530/bems.5.2.9

Article Available online

http://www.bemsreports.org

Copyright

(c) 2019 Phcog.Net. This is an open-access article distributed under the terms of the Creative Commons Attribution 4.0 International license. 


\section{MATERIALS AND METHODS}

Data from the REDUCE-IT trial recently published and presented in conferences was used for this study analysis.- ${ }^{7-9,12}$ Specifically the year wise 5 year cohort data on 1) All-cause mortality in USA subgroup in secondary prevention, 2) All-cause mortality in USA subgroup in Primary prevention, 3) All-cause mortality in USA subgroup, 4) Key secondary endpoints in USA subgroup, 5) Primary endpoint in USA sub group, 6) Primary composite endpoint in Reduce IT and 7) Secondary composite endpoint in Reduce IT were analysed. All the graphs analysed in this study were standard XY graph with adverse event rates represented in the $\mathrm{Y}$ axis and the time (in years) represented in $\mathrm{X}$ axis.

To assess the chrono-pharmacological impact of Icosapent Ethyl therapy, the year wise slope of the placebo and Icosapent Ethyl graphs was estimated using a digital imaging analysis software (NIH ImageJ). The change in the slope of Icosapent Ethyl data was calculated as the percent change from that of the placebo group. A higher value of the percentage change in the slope reflects a lower slope of the Icosapent Ethyl group and hence a lower rate of adverse events in this group compared to the placebo group. While a lower value of the percentage change in the slope reflects a higher slope of the Icosapent Ethyl group and hence a higher rate of adverse events in this group compared to the placebo group.

\section{RESULTS}

The results from this study analysis is presented in Figure 2 and 3. The year wise slope of the Icosapent Ethyl group considerably differed from the placebo group in all the study cohorts (Figure 2). In most of the study cohorts (except the primary and secondary composite endpoint in Reduce IT) there was a decline in the percent change in slope of the Icosapent Ethyl curves post year 5, indicating a higher rate of patients with adverse events in this group (Figure 3).

In all the study cohort's improvement was observed in years 2 and 3 following Icosapent Ethyl administration, however there was a decline in the percent change in slope of the Icosapent Ethyl curves between year 3 to year 4 (except for All-cause mortality in USA subgroup in secondary prevention and All-cause mortality in USA subgroup) (Figure 3), again indicating a higher rate of patients with adverse events in this group at Year 4 of Icosapent Ethyl therapy. This setback with adverse event rates in the year 4 was transiently corrected in year 5 prior to its decline post year 5 (Figure 3).

\section{DISCUSSION}

This study provides a novel approach to analyse the pharmacological efficacy of drugs used to treat chronic diseases. The current norm of assessing the drug efficacy based on a cumulative impact may be unable to identify transient adverse effects of the drug due to cumulative accumulation or may undermine the optimal outcomes achievable from therapeutically efficacious drug. To address this gap in this study, year wise efficacy of the drug was assessed based on comparing the slope of the annual curve of the therapeutic compound with that of the placebo group. The advantage of this approach was the feasibility to assess the year wise performance of the therapeutic compound, which is specifically helpful for drugs used on a chronic time frame.

Despite the cumulative efficacy of the Icosapent Ethyl therapy in reducing the cardiovascular adverse event rates, ${ }^{7,9}$ this study results indicated that the efficacy of Icosapent Ethyl therapy which was progressively better in the first 3 years of the therapy declined and in fact was worse than the placebo group in between years 3 to 4 of Icosapent Ethyl therapy. This study analysis indicated a higher rate of the cardiovascular adverse events in Icosapent Ethyl therapy between years 3 to 4 than the placebo group. Although the therapeutic setback observed in the years 3 to 4

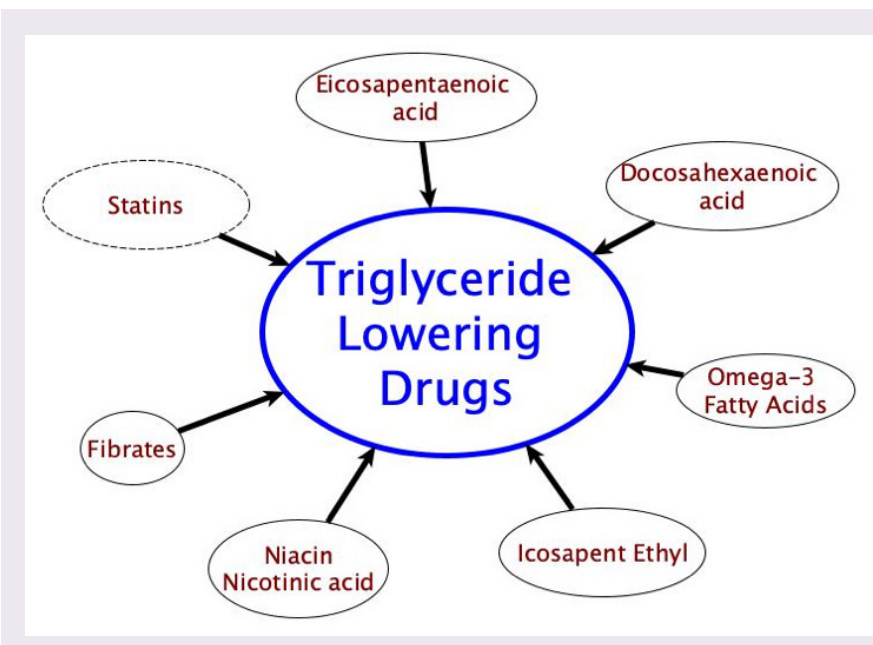

Figure 1: Drugs used for the treatment of hyper triglyceridemic.

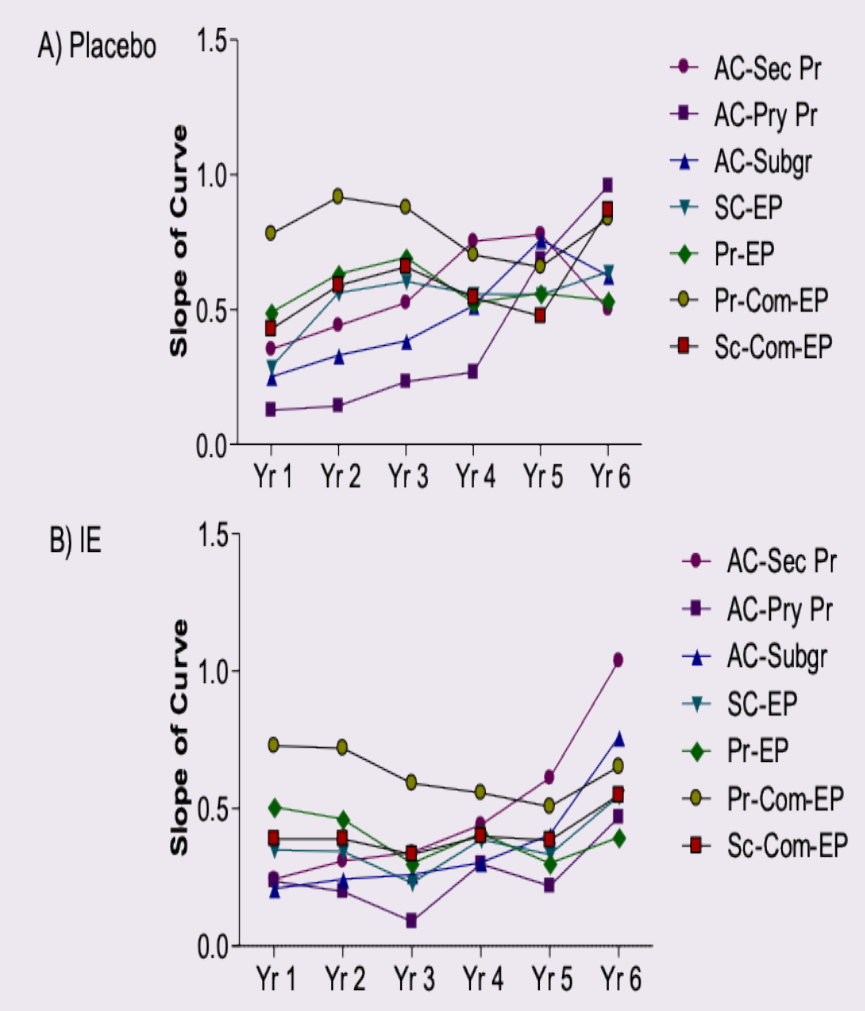

Figure 2: Chrono pharmacological assessment of slope of cardiovascular adverse effects curves in placebo (A) and Icosapent Ethyl (B) administered group.

Abbreviations used: All-cause mortality USA group Secondary prevention (AC-SEC-Pr), All-cause mortality USA group Primary prevention (AC-Pry-Pr), All-cause mortality USA subgroup (AC-Subgr), Key secondary endpoints USA subgroup (SC-EP), Primary end point USA sub group (Pr-EP), Primary Composite Endpoint Reduce IT (Pr-ComEP), Secondary Composite Endpoint Reduce IT (Pr-Com-EP)

was only transient and improved until year 5, a second setback with the Icosapent Ethyl therapy was observed post year 5. The reasons for observing such therapeutic setbacks is unclear but can be speculated due to possible tissue specific accumulation of the drug. It may also be likely that if such setbacks were not observed, then probably the cumulative therapeutic efficacy of the Icosapent Ethyl therapy over 5 years would be 


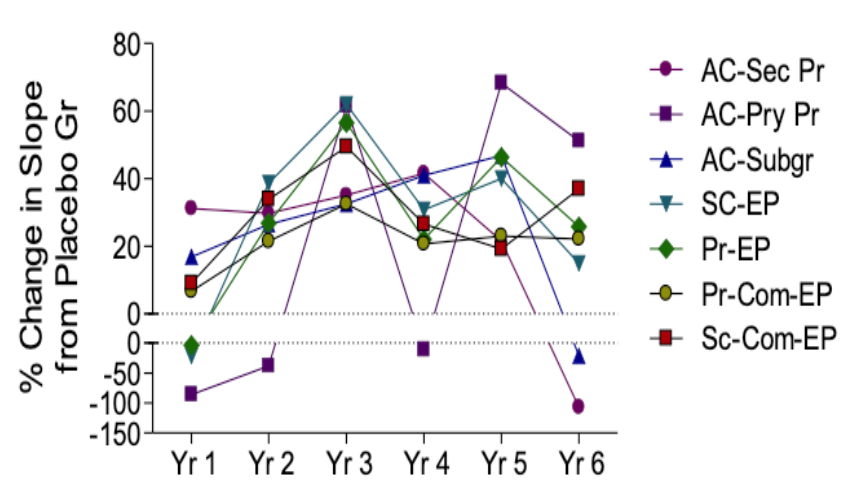

Figure 2: Chrono pharmacological assessment of percentage change in the slope of cardiovascular adverse effects curves in Icosapent Ethyl therapy administered group over to that of the placebo group.

Abbreviations used: All-cause mortality USA group Secondary prevention (AC-SEC-Pr), All-cause mortality USA group Primary prevention (AC-Pry-Pr), All-cause mortality USA subgroup (AC-Subgr), Key secondary endpoints USA subgroup (SC-EP), Primary end point USA sub group (Pr-EP), Primary Composite Endpoint Reduce IT (Pr-Com-EP), Secondary Composite Endpoint Reduce IT (Pr-Com-EP).

much superior to that reported in the REDUCE-IT trial. One strategy to address such setbacks will be by adopting interrupted therapeutic regimen i,e., in case of Icosapent Ethyl therapy putting the patients on the drug for first 3 year's followed by an appropriate withholding period and then restarting the Icosapent Ethyl therapy may be therapeutically and financially beneficial ${ }^{16}$ compared to patients taking the Icosapent Ethyl on a continuous basis.

This study identifies an important and perhaps a novel aspects of chrono pharmacology based approach to recommend drug dosing regimen to achieve optimal therapeutic efficacy and patient safety. Besides achieving optimal therapeutic efficacy, chrono pharmacological evaluations will also be helpful in improving cost effectiveness of drug use. Chrono pharmacological modelling of the drug dosage regimen may also be applicable in personalised medicine. Future studies evaluating the chrono pharmacodynamics and chrono pharmacokinetics of all the drugs used for the therapy of chronic disease conditions are warranted to better understand the application of the chrono pharmacology in recommending dosing regimen to achieve optimal therapeutic outcomes.

\section{ACKNOWLEDGEMENT}

Research support from University College Dublin-Seed funding/Output Based Research Support Scheme (AHSK), Royal Society-UK (AHSK) and Stemcology (AHSK) is acknowledged.

\section{CONFLICT OF INTEREST}

No conflict of interest to declare.

\section{ABBREVIATIONS}

AC-SEC-Pr: All-cause mortality USA group Secondary prevention; ACPry-Pr: All-cause mortality USA group Primary prevention; AC-Subgr:
All-cause mortality USA subgroup; SC-EP: Key secondary endpoints USA subgroup; Pr-EP: Primary end point USA sub group; Pr-Com-EP: Primary Composite Endpoint Reduce IT; Pr-Com-EP: Secondary Composite Endpoint Reduce IT; REDUCE-IT: Reduction of Cardiovascular Events with Icosapent Ethyl Intervention Trial.

\section{REFERENCES}

1. Toth PP, Philip S, Hull M, Granowitz C. Elevated Triglycerides (>/=150 mg/dL) and High Triglycerides (200-499 mg/dL) Are Significant Predictors of New Heart Failure Diagnosis: A Real-World Analysis of High-Risk Statin-Treated Patients. Vasc Health Risk Manag. 2019;15:533-8.

2. Sultani $\mathrm{R}$, et al. Elevated Triglycerides to High-Density Lipoprotein Cholesterol (TG/HDL-C) Ratio Predicts Long-Term Mortality in High-Risk Patients. Heart Lung Circ. 2019;S1443-9506(19):30334-8. doi: 10.1016/j.hlc.2019.03.019. [Epub ahead of print].

3. Jorgensen AB, et al. Genetically elevated non-fasting triglycerides and calculated remnant cholesterol as causal risk factors for myocardial infarction. Eur Heart J. 2013;34(24):1826-33.

4. Hallen J, Sreeharan N. Development of triglyceride-lowering drugs to address residual cardiovascular risk: Strategic and clinical considerations. Eur Heart J Cardiovasc Pharmacother. 2018;4(4):237-42.

5. Maki KC, Dicklin MR. Do triglyceride-lowering drugs decrease risk of cardiovascular disease?. Curr Opin Lipidol. 2017;28(4):374-9

6. Feingold KR, Grunfeld C. In Endotext. 2000.

7. Boden WE, et al. Profound reductions in first and total cardiovascular events with icosapent ethyl in the REDUCE-IT trial: Why these results usher in a new era in dyslipidaemia therapeutics. Eur Heart J. 2019;1-10. doi: 10.1093/eurheartj/ ehz778. [Epub ahead of print])

8. Bhatt DL, et al. Effects of Icosapent Ethyl on Total Ischemic Events: From Reduce-It. J Am Coll Cardiol. 2019;73(22):2791-802.

9. Bhatt DL, et al. Cardiovascular Risk Reduction with Icosapent Ethyl for Hypertriglyceridemia. N Engl J Med. 2019;380(1):11-22.

10. Ballantyne CM, et al. Icosapent ethyl (eicosapentaenoic acid ethyl ester): Effects on remnant-like particle cholesterol from the MARINE and ANCHOR studies. Atherosclerosis. 2016;253:81-7.

11. Fares H, Lavie CJ, DiNicolantonio JJ, O'Keefe JH, Milani RV. Icosapent eth$\mathrm{yl}$ for the treatment of severe hypertriglyceridemia. Ther Clin Risk Manag. 2014; 10:485-92.

12. Bhatt $\mathrm{DL}$, et al. Reduction in First and Total Ischemic Events with Icosapent Ethyl across Baseline Triglyceride Tertiles. J Am Coll Cardiol. 2019;74(8):1159-61.

13. Nelson JR, Wani O, May HT, Budoff M. Potential benefits of eicosapentaenoic acid on atherosclerotic plaques. Vascul Pharmacol. 2017;91:1-9.

14. Mason RP, Jacob RF, Shrivastava S, Sherratt SCR, Chattopadhyay A. Eicosapentaenoic acid reduces membrane fluidity, inhibits cholesterol domain formation and normalizes bilayer width in atherosclerotic-like model membranes. Biochim Biophys Acta. 2016;1858(12):3131-40.

15. Miller M, et al. Effects of Icosapent Ethyl (Eicosapentaenoic Acid Ethyl Ester) on Atherogenic Lipid/Lipoprotein, Apolipoprotein and Inflammatory Parameters in Patients with Elevated High-Sensitivity $\mathrm{C}$ - reactive protein (from the ANCHOR Study). Am J Cardiol. 2019;124(5):696-701.

16. Ademi Z, Ofori-Asenso R, Zomer E, Owen A, Liew D. The cost-effectiveness of icosapent ethyl in combination with statin therapy compared with statin alone for cardiovascular risk reduction. Eur J Prev Cardiol. 2020. https://doi. org/10.1177/2047487319896648.

17. Dreesen E, Gils A. Pharmacodynamic Monitoring of Biological Therapies in Chronic Inflammatory Diseases. Ther Drug Monit. 2019;41(2):131-41.

18. Sulli G, Manoogian ENC, Taub PR, Panda S. Training the Circadian Clock, Clocking the Drugs and Drugging the Clock to Prevent, Manage and Treat Chronic Diseases. Trends Pharmacol Sci. 2018;39(9):812-27.

19. Onder $G$, et al. Accounting for frailty when treating chronic diseases. Eur J Intern Med. 2018;56:49-52.

20. Al-Azzam SI, Alzoubi KH, Alefan Q, Alzayadeen RN. Evaluation of the types and frequency of drug-related problems and the association with gender in patients with chronic diseases attending a primary health care center in Jordan. Int Health. 2016;8(6):423-6.

21. Angulo J, EIAssar M, Rodriguez-Manas L. Frailty and sarcopenia as the basis for the phenotypic manifestation of chronic diseases in older adults. Mol Aspects Med. 2016;50:1-32

Cite this article : Kumar AHS, George M. Evaluating the Chrono-pharmacology of Icosapent Ethyl to Assess its Therapeutic Efficacy in Reducing Hypertriglyceridemia Associated Cardiovascular Events. BEMS Reports. 2019;5(2):26-8. 